\title{
Um ambiente de comunidade virtual baseado em objetos de aprendizagem para apoiar a aprendizagem em museus
}

\author{
Ana Carolina Bertoletti-De-Marchi** \\ Cristiane Durigon Testa * \\ Antônio Carlos da Rocha Costa *
}

\begin{abstract}
Resumo: Os museus produzem e disponibilizam uma grande variedade de informações. Uma maneira de tornar essas informações disponíveis para o público é através do suporte a interoperabilidade de dados. Várias propostas para isso estão sendo elaboradas e implementadas, através do uso de metadados descritivos. Como a meta deste trabalho é promover a aprendizagem em museus, este artigo pretende apresentar um ambiente de comunidade virtual de aprendizagem baseado em objetos de aprendizagem com a inclusão de ferramentas para suportar a aprendizagem em museus. $\mathrm{O}$ ambiente pretende ajudar estudantes e visitantes casuais a fazerem um melhor uso das informações disponíveis nos museus.
\end{abstract}

Palavras-chaves: objetos de aprendizagem, comunidades virtuais e aprendizagem informal em museus.

The Virtual Community Environment based on learning objects to support the learning in museums.

Abstract: Museums produce and make available a large variety of information. A way to make that information widely available for the general public is to support the interoperability of such data. Various proposals for such support are being elaborated and implemented, through the use of the descriptive metadata. Since the goal of the present work is to promote learning in museums, this paper intends to present a virtual community learning environment based on learing objects will enhance the tools available for the support of learning in museums. The environment intends to help regular students and casual visitors to make the best of the information available in museums.

Keywords: learning objects, virtual community and informal learning in museums.

\section{Introdução}

Os museus são ambientes educacionais que estão contribuindo para o acréscimo de novas fontes de materiais digitais na Internet. Eles se constituem em um espaço educativo informal que complementa a aprendizagem, tanto de alunos como do público em geral. Segundo Trant et al. (2002), essa nova diversidade de fontes eletrônicas oferece muitas vantagens aos museus, como: a frequiente atualização, a possibilidade de suportar múltiplos usuários, o acesso sem considerar as horas em que está aberto, a oportunidade de aperfeiçoamento por parte dos leitores e o acesso a informações por pesquisadores.

\footnotetext{
" Artigo elaborado a partir da proposta de tese de doutorado no PGIE e com o apoio do CNPq, edital MCT/SECIS/CNPq $\mathrm{n}^{\circ} 07 / 2003$

"Professora, doutoranda em Informática na Educação (PGIE/UFRGS), professora e pesquisadora da UPF, carolina@upf.br. http://vitoria.upf.br/ carolina

*** Bolsista de Iniciação Científica da FAPERGS e aluna do Curso de Ciência da Computação/UPF. 57547@lci.upf.br.

"*t** Professor, Doutor em Ciência da Computação, professor e pesquisador da UCPel, rocha@atlas.ucpel.tche.br V. $3 \mathrm{~N}^{\circ} 1$, Maio, 2005
} 
No entanto, atualmente, a Internet não é vista apenas como um repositório de informações, mas também como um meio pelo qual as pessoas podem participar de grupos sociais medidos por computadores, conhecidos como Comunidades Virtuais (CVs). Nas CVs as pessoas utilizam diferentes formas de comunicação para discutir assuntos de interesse em comum, promover discussões intelectuais, trocar conhecimentos e propiciar meios alternativos de aprendizagem.

Já que o foco de estudo deste trabalho é a informática educativa nos museus de ciências, partimos do pressuposto que a informação e a comunicação são essenciais para o processo de aprender no contexto informal dos museus. Baseados nisso, concordamos com Carter (1996), quando afirma que a missão fundamental dos museus é colecionar e conservar os objetos relacionados com a sua natureza e cultura, mas a informação adquirida terá mais valor se for passada à comunidade mundial. Esse processo de comunicação é definido pelo autor, como uma atividade educacional do museu. Quando a comunicação se estabelece com a intenção de fazer o visitante conhecer uma informação, a mesma é chamada por Blanco (1997, p.85) de estratégia comunicativa, onde podemos dizer que existe a intenção de ensinar.

No contexto deste trabalho, a comunicação é parte integrante da aprendizagem proporcionada dentro das comunidades virtuais. Já a informação é vista como sendo os recursos de aprendizagem oferecidos pelos museus, os quais denominamos de objetos de aprendizagem.

Este trabalho tem como objetivo mostrar que é possível aprimorar a aprendizagem informal em museus através da construção de comunidades virtuais de aprendizagem, com acesso a repositórios de objetos de aprendizagem e ferramentas interativas para apoio a atividades didáticas. Queremos mostrar que a construção, em museus, de comunidades virtuais de aprendizagem baseadas em objetos de aprendizagem, além de ser uma inovação na área, contribui significativamente no apoio a aprendizagem informal naqueles ambientes.

Com base no exposto acima, pretendemos, com este trabalho, aumentar ainda mais a contribuição dos museus nos processos de aprendizagem de estudantes e da sociedade em geral. Além de propiciar a instalação e o fortalecimento dos museus, promovendo sua expansão a nível mundial, através da difusão e popularização da cultura científico-tecnológica por meio de um ambiente que possui a junção de várias tecnologias, as quais irão propiciar mais uma forma de comunicação dentro dos museus para apoiar a aprendizagem informal em ambientes virtuais.

Neste sentido, iremos apresentar, primeiramente, uma fundamentação teórica e, após, como estão dispostos os objetos de aprendizagem que estão cadastrados no repositório, sua recuperação e reutilização através dos métodos de pesquisa. Por fim, o artigo irá descrever a arquitetura geral do ambiente de comunidade virtual.

\section{Museu: Um ambiente de aprendizagem informal}

A definição de museu mudou muito ao longo dos tempos, atualmente é reconhecido que sua função não se limita ao ato de coletar, restaurar e expor objetos que compreendem o acervo. A pesquisa, a divulgação, a socialização do conhecimento, tornaram-se, com o passar dos tempos, elementos determinantes nas funções sociais dos museus. Cada vez mais, o objetivo dos museus é ajudar as pessoas a compreenderem o mundo que as cerca, visando atingir, através disto, tanto a população num sentido global, como especialmente orientando suas ações para crianças e jovens em idade escolar e, por consequiência, a professores que com eles atuam.

$\mathrm{O}$ museu prove um ambiente de aprendizagem ideal, como espaço paralelo à educação formal, pode contribuir significativamente com o ensino, pois pode ser visto como um lugar estimulante e aberto, visto até como um espaço de encontro, 
convivência, investigação e confrontação das diversidades de linguagens. Independente de sua filosofia e área de atuação, todo o museu transmite ao público uma mensagem, educando através da cultura material qualquer pessoa.

O papel educativo que os museus apresentam, tem sido um dos fatores mais analisados e ressaltados desde sua renovação. Independente do ponto de vista que se considere, as ações dos museus só se justificam social e culturalmente em função de seu público. Segundo Fernández (1993, p.257), esta é a razão pela qual a maior parte das investigações e experiências desenvolvidas nos últimos anos está focando a dimensão pedagógica dos museus.

Um museu é uma instituição a serviço da sociedade. Seu papel educativo vem gradualmente assumindo posições de destaque no mundo da museologia. A Educação é um processo contínuo e ininterrupto que instrumenta o indivíduo para uma função na sociedade e constitui também processo de socialização. Entre os objetivos fundamentais dos museus a educação precede a todos os demais.

O papel educacional do museu é bem amplo já que pode ser utilizado por todos os setores da população ou grupos especializados, aos quais ele tem por meta servir. $\mathrm{O}$ museu deve oferecer a comunidade em geral, bem como a indivíduos ou grupos específicos, oportunidade de envolvimento ativo para que apóiem seus objetivos. Segundo Cabral (2002), os objetivos da educação nos museus são os mesmos da escola “...espaço para a comunicação, informação, aprendizado, a relação dialética e dialógica educando/educador, a construção da cidadania, o entendimento do que seja identidade, mas a educação em museus deverá operar promovendo atividades baseadas em metodologias próprias que permitam a formação de um sujeito histórico-social que analisa criticamente, recria e constrói a partir de um referencial que se situa nos seus objetos tangíveis e intangíveis."

É indispensável que os museus estabeleçam uma dinâmica diferente da presente em sala de aula, fomentando assim uma aprendizagem informal, com características distintas da oferecida no sistema formal. Conforme Cazelli (1998), os professores ponderam que a visita ao museu é extremamente proveitosa por razões distintas como: contemplar a escola contribuindo para uma melhor sedimentação dos conteúdos trabalhados; motivar para a posterior abordagem de diferentes conteúdos programáticos; compensar a carência de recursos didáticos e laboratoriais da escola; criar oportunidade para uma relação entre a teoria e a prática. Segunda a autora: "Mudar a atitude pedagógica perante o museu, é uma desconstrução que exige mais do que uma capacitação de algumas horas e domínio de certos conteúdos. Demanda o domínio dos bastidores do Museu, de uma pedagogia distinta da escola, e a compreensão de que se trata de outra forma de mediar a aprendizagem, visto que os museus são meios de comunicação onde o olhar, o tocar, o andar, o emocionar-se, pressupõe liberdade de escolha, estar à vontade e descomprometido com qualquer terminalidade."

A maioria das visitas a museus é uma atividade de livre escolha, com motivação intrínseca que favorece a aprendizagem ao longo da vida e de uma forma casual. Assim sendo, vemos a aprendizagem informal do museu como uma aprendizagem casual, que segundo Mansur e Moretto (2000, p.14), “...é quase espontânea, surge naturalmente da interação entre as pessoas e o ambiente em que vivem. Ela se dá pela convivência social, pela observação, leituras, conversas, etc."

Os museus são uma alternativa para construir conhecimento, despertando a investigação e desenvolvendo a capacidade de solucionar problemas. Vê-se que o papel educacional dos museus é muito amplo e realmente é a sua missão principal. Os museus têm condições de desempenhar uma importante função para o crescimento do nível educacional da população, completando a aprendizagem tanto dos alunos que ainda freqüentam as escolas, como do público em geral. 
Adicionalmente, apoiados pelo avanço da tecnologia, os museus podem fornecer uma grande gama de educação interativa através de seus experimentos, os quais instigam o visitante a investigar usando ferramentas cientificas e instrumentos que podem o encorajar a observar, procurar relações e fazer suas próprias conclusões.

\section{Propostas para o uso de objetos de aprendizagem em museus}

Os objetos de aprendizagem estão sendo amplamente utilizados por educadores como uma abordagem eficiente para criar ricas e significantes experiências de aprendizagem.

Os museus têm adotado abordagens baseadas em coleções no desenvolvimento de objetos de aprendizagem, usando itens individuais da história de suas coleções como âncora para projetos de experiências on-line, a fim de engajar estudantes na exploração de temas chave. Cada objeto de aprendizagem é projetado para ser uma experiência sozinha, mas também podem ser organizados em uma seqüência de muitos objetos refletindo diferentes pontos de vista.

Para apoiar a aprendizagem informal em museus, os objetos de aprendizagem podem ser utilizados das mais diversas formas, considerando um público bastante diversificado em contextos distintos.

Podemos iniciar a analise de propostas de uso de objetos de aprendizagem em museus pensando nas distinções com relação ao público alvo, onde diferenciamos o visitante on-line do visitante do museu físico. Chadwick (1999), em seus estudos sobre as diferenças entre os visitantes, concluiu que todos os usuários buscam em um site de museu um ambiente informal de aprendizagem, isto é, um recurso extra de aprendizado, principalmente os grupos, pois procuram o site para pesquisar e aprender.

De acordo com o contexto acima, os objetos de aprendizagem podem ser utilizados por ambos visitantes para: realizar uma pesquisa; aprofundar conhecimentos; ser utilizado na construção de um curso; servir como material de apoio; entre outros.

Vários museus ao redor do mundo estão adotando os OAs em sua realidade. No The National Museum of Australia os learning objects são utilizados para $o$ desenvolvimento de multimídias educacionais on-line para uso em sala de aula utilizando os recursos e coleções do museu. Essa representação digital das coleções acarreta mudanças na representação dos objetos pertencentes as mesmas para uso no espaço interativo on-line. Essas mudanças estão sendo estudadas por Payne e Peacock (2004).

Observa-se que o uso de OAs em museus é muito amplo e atinge os mais diversos públicos, desde o público em geral, até alunos e professores do sistema formal.

No momento em que os museus construírem um repositório de OAs, os mesmos estarão ampliando sua missão com o público, oferecendo mais um serviço pronto a auxiliar na construção do conhecimento, seja no ensino formal, não-formal ou informal. Devemos aproveitar todo o conhecimento que é gerado pelas equipes de museus, não o deixando restrito ao mundo museal.

\section{Comunidades Virtuais de Aprendizagem}

Uma CV de aprendizagem é aquela voltada a promover um ambiente que favorece a construção de conhecimento, onde seus membros estão relacionados com objetivos em comum de aprendizagem. Essas comunidades podem promover a aprendizagem seguindo uma estrutura formal ou informal. Quando mencionamos a forma formal, estamos considerando que a comunidade irá se formar com base em uma 
estrutura física real, onde encontramos a figura do professor como facilitador e do aluno como ativo na construção de seu conhecimento. Este tipo de comunidade é bastante descrita em Pallof e Pratt (2002), onde a tecnologia é vista como uma forma de construir uma sala de aula virtual.

Entretanto, este trabalho irá se deter nas características obtidas por meio das comunidades de aprendizagem informais, as quais denominamos aqui como sendo aquelas que, para sua formação, contam com os interesses pessoais dos membros de modo a definir uma rede de relacionamento auto-organizada, proporcionada através da comunicação interativa, visando objetivos comuns que os levem a aprendizagem contínua e permanente.

Para a definição do termo informal, nos baseamos na abordagem de Castells (apud Schlemmer, 2001) quando fala do modo informacional de desenvolvimento presente nas CVs que contribuirão para o surgimento de uma nova economia do conhecimento. Seguiremos o mesmo posicionamento de Souza (2000) onde especifica que "...o aprendizado ocorre mesmo à parte de programas e estruturas formais que objetivem-no. Mesmo em listas de discussão que não possuem um corpo formal de objetivos a alcançar, ou em interações exclusivamente 'sociais' em comunidades virtuais, ocorre uma espécie de aprendizado contínuo e de grande valia, que atinge os participantes de forma heterogênea."

A "espécie de aprendizado" ressaltada pelo autor será aqui estabelecida como uma aprendizagem construtivista autodirigida, baseada na colaboração e na autoorganização, contando, para isso, com a participação ativa de todos os participantes. A participação através da troca de contribuições inicia os processos dialéticos de proposições e réplicas, que propiciam a construção do conhecimento.

Segundo Pallof e Pratt (2002, p.39), a aprendizagem autodirigida habilita os membros de uma comunidade a seguir interações onde quer que elas os levem, não sendo dependentes da figura do professor. Jonassen et al (Pallof e Pratt, 2002, p. 39), discutem o resultado dessa forma de aprendizagem e observam que "...a facilitação proporcionada pelos ambientes de aprendizagem que estimulam a construção de sentido pessoal, bem como a construção social do conhecimento e do significado por meio de interações com outras comunidades de alunos, é preferível às intervenções do professor, que controlam a seqüência e o conteúdo da instrução." O processo de aprendizagem autodirigida está centrado nos membros da comunidade, com os mesmos decidindo o ritmo e a direção de todo o processo.

No entanto, para que a aprendizagem autodirigida dos participantes da comunidade seja bem sucedida, envolvendo os mesmos ativamente na produção do conhecimento, existem alguns papéis que devem ser desempenhados. Tais papéis foram adaptados de Pallof e Pratt (2002, p.109) para as comunidades de aprendizagem informais.

- Produção de conhecimento: os participantes são responsáveis por buscar soluções para os problemas inerentes a área de conhecimento do foco de estudo, bem como elevar essas soluções a um nível mais complexo.

- Colaboração: os participantes devem trabalhar juntos na produção de níveis de compreensão mais complexos e na avaliação crítica do material de estudo. Eles sempre devem compartilhar com os demais participantes materiais adicionais, informações relevantes, etc..

- Gerenciamento do processo: o participante ativo deve seguir diretrizes mínimas e interagir entre os demais membros, manifestando sempre sua opinião sobre o que está ocorrendo na CV. Deste modo, ele estará adquirindo responsabilidades e estará diretamente envolvido em seu processo de aprendizagem. 
Esses papéis funcionam como requisitos básicos para que os participantes da CV consigam realmente usufruir todos os benefícios de aprendizagem oferecidos.

Não basta o ambiente apresentar todas as características para promover a aprendizagem sem que os participantes desempenhem realmente um papel ativo durante todo o processo. Não é apenas o ambiente como um todo, e sim a participação dos membros que sustentam e suportam muitas interações para que a aprendizagem ocorra. É durante esses processos de interação que os participantes ativos constroem e expressam competências.

Adicionalmente, Costa (2004) aponta três pontos distintos a se considerar na construção de uma comunidade virtual voltada para a aprendizagem:

- repositório de recursos didáticos: serve para disponibilizar o material a ser trabalhado pelos participantes da comunidade, os quais podem ser vistos como os objetos de aprendizagem;

- ambiente de apoio ao ensino-aprendizagem: serve para organizar as atividades didáticas; e

- sistema de gerência da comunidade: serve para organizar as interações sociais na comunidade.

Nesta seção discutimos aspectos relevantes na construção de comunidades virtuais relacionados com o ponto 3 , levando em consideração como as atividades podem ser organizadas e mencionando a importância do repositório na constituição das mesmas. Cabe ressaltar que neste trabalho iremos nos deter nos pontos 1 e 2 .

\section{0 ambiente}

A necessidade de envolver cada vez mais o visitante do museu e torná-lo cúmplice da experiência vivenciada, acabando com o destinatário passivo do discurso expositivo que se estabelecia unilateralmente, juntamente com a crescente vontade de ampliar o canal comunicativo dos museus, oferecendo ao público acesso a uma vasta quantidade de informações produzidas e mantidas por suas equipes, justificam o objetivo principal deste trabalho.

Estamos desenvolvendo um ambiente de comunidade virtual para apoiar a aprendizagem informal em museus, através do acesso as informações, as quais estão depositadas em um repositório na forma de objetos de aprendizagem. Os OAs, com todas as suas características, favorecem a expansão comunicativa do museu, uma vez que possibilitam a criação de materiais didáticos simples, pequenos e que podem ser facilmente utilizados fora do ambiente do museu, em sala de aula, por pesquisadores e pelo público em geral.

Neste sentido, de modo a facilitar a compreensão do ambiente de comunidade virtual, o mesmo será apresentado em dois módulos distintos e interoperantes: sistema de gerência de repositório de recursos didáticos; e ambiente de apoio ao ensinoaprendizagem.

A figura 1 ilustra a arquitetura geral do ambiente que visa disponibilizar para consulta os objetos de aprendizagem de museus para acesso virtual a um público em geral. Tal consulta considera as informações semânticas dos objetos, permitindo sua fácil recuperação, considerando os objetivos educacionais do professor, aluno e público em geral. 


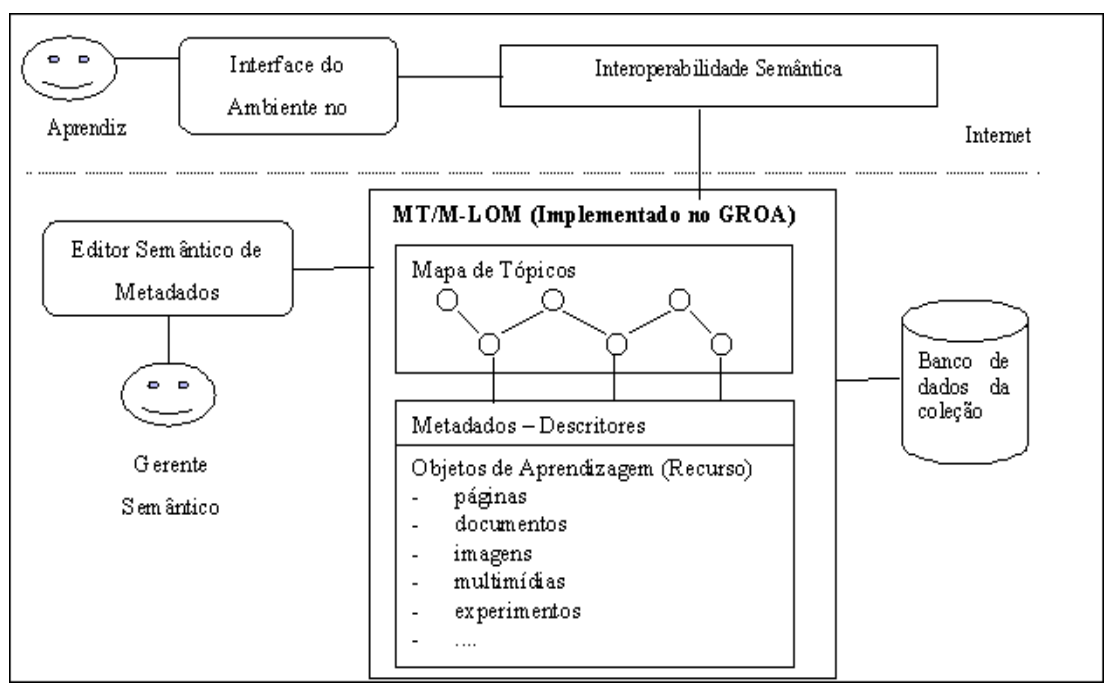

Figura 1 - Estrutura do Ambiente

\subsection{Sistema de gerência de repositório de recursos didáticos (MT/M-LOM)}

O MT/M-LOM é o sistema que gerencia todo o repositório de recursos de aprendizagem do museu, onde serão disponibilizados todos os objetos de aprendizagem disponíveis. Ele foi implementado no Sistema de Gerência de Repositórios de Objetos de Aprendizagem (GROA) e seguindo as extensões propostas com o M-LOM (Bertoletti-De-Marchi 2004).

O GROA (Miranda 2004) tem como objetivo gerenciar o armazenamento de OAs via Internet, disponibilizando recursos de armazenamento, gerenciamento, indexação e estruturação, que suporte a inclusão de novos padrões e possibilite a consulta e recuperação remota dos objetos armazenados. Para o armazenamento dos OAs, o GROA utiliza o esquema de dados conceitual LOM, produzido pelo padrão IEEE, que define a estrutura de uma instância de metadados para um OA.

Para permitir a recuperação semântica, os OAs são formados pelo recurso e o metadado descritor, que segue as especificações do M-LOM. Estes descritores são definidos pelo gerente semântico, o qual é representado pelo funcionário do museu conhecedor das coleções e artefatos em exposição. Tal conhecimento é imprescindível para o cadastro dos OAs, uma vez que deve haver um controle muito rígido para que a grande maioria dos elementos de dados seja preenchida no momento do cadastro. $\mathrm{O}$ preenchimento inadequado ou incompleto de tais informações acaba por gerar uma base pouco eficiente no que se refere à recuperação dos dados. Atualmente, o ambiente já possui cerca de 60 objetos cadastrados, entre multimídias, artigos, jogos educativos, etc.

Adicionalmente, além da busca através dos metadados, o ambiente também proporciona uma busca hierárquica por meio dos mapas de tópicos, que são definidos pelo gerente semântico juntamente com os responsáveis pelas coleções didáticas e científicas do museu. Tal busca assemelha-se a uma varredura em uma árvore onde os nodos folhas estão diretamente conectados aos elementos de dados do M-LOM. Os mapas de tópicos podem auxiliar o gerente semântico a entrar com os dados em uma forma semântica válida. Os mapas de tópicos também auxiliam o visitante a recuperar as informações. 
Com isto, o ambiente permite a busca bilateral, proporcionando ao visitante duas formas distintas de encontrar o objeto de aprendizagem desejado.

A implementação do MT/M-LOM proposto neste trabalho está baseada no GROA, mas pode ser implementado em qualquer sistema que trabalhe com objetos de aprendizagem e mapas de tópicos.

A relação bilateral entre o mapa de tópicos e o metadado descritor, proporciona ao visitante duas formas distintas de buscar o recurso desejado nas bases de dados do museu, facilitando sua pesquisa, tornando-a mais eficiente, precisa e rápida. Além disso, estaremos favorecendo diferentes perfis dos visitantes: os que preferem uma busca por palavras-chave e os que aderem à navegação hierárquica (indexada) para encontrar a informação desejada.

Todo o cadastro de OAs ocorre por meio de uma interface implementada no ambiente Zope, o qual também serve com meio para que o visitante busque as informações desejadas na base. O cadastro se inicia com o preenchimento do metadado descritor do recurso, e, a seguir, é definida a taxonomia, onde é feito o relacionamento com o mapa de tópico já definido pelo administrador, conforme necessidade do ambiente onde foi desenvolvido o repositório de objetos de aprendizagem.

A pesquisa e recuperação dos OA por der feita pelo método de: busca direta, titulo, descrição ou palavras-chave.

\subsection{Ambiente de apoio ao ensino-aprendizagem}

$\mathrm{O}$ ambiente de apoio ao ensino-aprendizagem serve para organizar as atividades didáticas, oferecendo aos visitantes on-line acesso a um conjunto de ferramentas de comunicação e desafios.

O objetivo é fornecer ferramentas que possibilitem que alunos e professores assumam papéis semelhantes, como ativos construtores dos significados de suas experiências, buscando desenvolver o pensamento crítico dos mesmos. Nosso desejo é que, com a utilização do ambiente, todos os visitantes sejam vistos como iguais, procurando minimizar ao máximo a grande diversidade da audiência existente nestes ambientes informais. Assim, utilizamos intensamente a experimentação, a problematização, a reflexão e a pesquisa, fazendo com que os visitantes possam construir suas próprias conexões com os objetos de aprendizagem, oportunizando diversos caminhos de aprendizagem, onde a interação entre os participantes é a peça fundamental para o sucesso do ambiente.

Desta forma, estaremos oferecendo um espaço de encontros e convivências, promovendo discussões intelectuais, troca de conhecimentos, compartilhamento de idéias, entre pessoas com objetivos semelhantes, que o fazem dirigidos apenas por seus interesses e necessidades. Com o desenvolvimento deste módulo, o usuário pode interagir através de pesquisas dos OA com os metadados cadastrados no repositório, proporcionando a reutilização dos $\mathrm{OA}$ e enriquecendo o repositório através de seus comentários e produções relacionado ao OA pesquisado. Além de trocar informações com outros usuários, proporcionando diálogos e discussões sobre o assunto.

$\mathrm{O}$ ambiente está sendo desenvolvido utilizando a linguagem $\mathrm{Php}$, com o auxilio do banco de dados PostgreSql e através deste fazendo uma ponte de ligação por ODBC com o ZOPE, plataforma no qual foi desenvolvido o GROA. Com isso, é possível que eles compartilhem informações e recursos sobre os OA cadastrados e as produções inseridas pelos usuários.

No ambiente estão sendo desenvolvidas ferramentas como chat, fórum, mural, produção, pesquisa, entre outros. Na produção o usuário pode cadastrar no ambiente todas as suas produções pessoais através de um descritor de metadado. Essas produções 
ficam armazenadas no repositório dos usuários, permitindo a consulta a todos os membros. Cabe ressaltar que tanto a produção, quanto os fóruns criados pelos usuários, devem ser relacionados ao mapa de tópicos do sistema para permitir que outros usuários facilmente encontrem os trabalhos produzidos ou as discussões realizadas.

As produções são incluídas no ambiente através do sistema de upload de arquivo. O usuário deverá preencher um pequeno descritor que define: tipo de arquivo, palavras-chave, assunto, título e data e hora do upload. Conseqüentemente, também pode ser executada pesquisa sobre essas produções, fazendo a recuperação destes arquivos.

A figura 2 ilustra a tela principal do ambiente no módulo de pesquisa, onde pode ser efetuada a busca pelo direta pelo OA, pelo mapa de tópicos ou no repositório de produções dos usuários. Já a figura 3 ilustra o mapa de tópicos do sistema, implementado no GROA, onde pode ser efetuada a busca dos OAs através da navegação na estrutura de árvore.

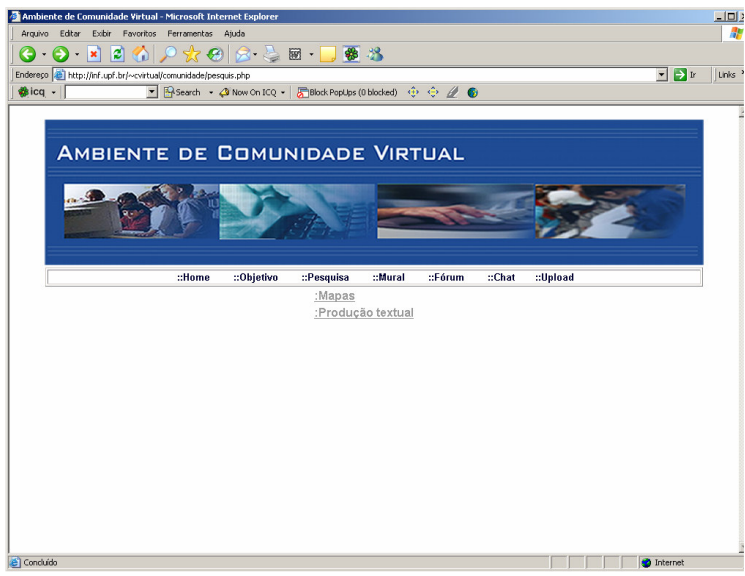

Figura 2 - Tela principal do ambiente

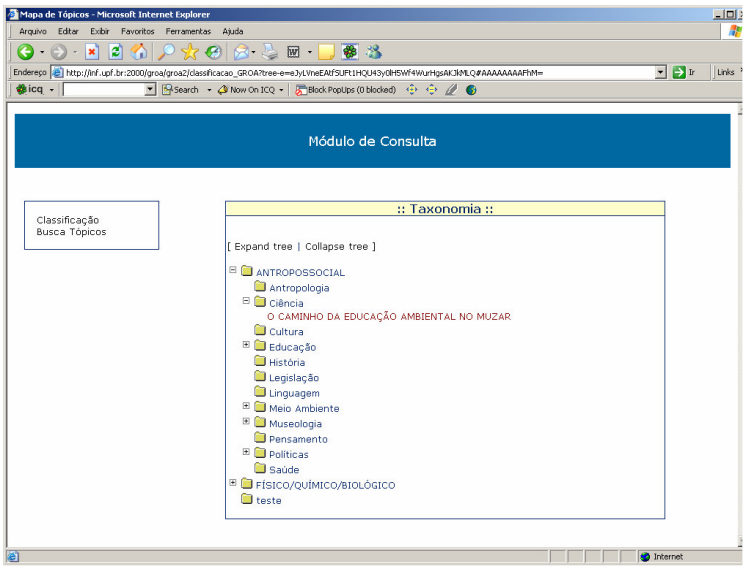

Figura 3 - Mapa de tópicos do Sistema

\section{Considerações finais}

A construção do ambiente descrito neste artigo tem relevância tanto a nível científico e tecnológico como educacional, trazendo contribuições tanto para a educação quanto para o desenvolvimento de sistemas para museus e para a pesquisa.

Com a informatização dos objetos de aprendizagem, os museus terão condições de melhorar a qualificação da linguagem museográfica, o que possibilitará facilidade de acesso à informação. Além de ter condições de transmitir as informações restritas ao mundo científico, para uma comunidade sedenta de informações sobre o Patrimônio Natural e sensibilizado para agir pela sua preservação.

Os museus, em vários momentos, são museu-escola ou museu-laboratório. Envolvendo seu visitante em uma exposição interativa ou observando a natureza em uma trilha interpretativa, vivenciando a aprendizagem acontecendo. Integrando a nova visão de museu ao movimento que a computação e a energia podem oferecer é possível disponibilizar com mais facilidade a informação. Informação esta, que muitas vezes fica omitida no íntimo da peça museológica, mas que interagindo com os atuais nobres meios de comunicação é possível transmitir conhecimento e influenciar a sensibilidade para a valorização dos Patrimônios Natural e Cultural. Neste momento, inserir a informatização dos objetos de aprendizagem de museus passa a ser, a principal forma de ampliar sua criação, trazendo para o museu a chance de atingir ainda mais a sociedade.

Com a implantação deste ambiente de busca bilateral estaremos propiciando a instalação e o fortalecimento institucional dos museus, promovendo sua expansão a 
nível mundial, bem como auxiliando na melhoria da qualidade do ensino das ciências. Além de promover a interoperabilidade de dados entre as instituições. Com o ambiente de comunidade virtual implantado, o museu terá uma parcela ainda maior de contribuição no processo de aprendizagem de jovens estudantes de todos os níveis escolares assim como da comunidade em geral, pois irá difundir e popularizar a cultura científico-tecnológica.

\section{Agradecimentos}

Agradecemos o Conselho Nacional de Desenvolvimento Científico e Tecnológico CNPq - pela aprovação do projeto, através do edital MCT/SECIS/CNPq n ${ }^{\circ}$ 07/2003 e a Fundação de Amparo a Pesquisa do Rio Grande do Sul (FAPERGS) pela concessão de uma bolsa de iniciação científica.

\section{Referências Bibliográficas}

Bertoletti-De-marchi, A. C. (2004). Um ambiente de comunidade virtual apoiada por objetos de aprendizagem para suporte à aprendizagem informal em musues. Proposta de tese de doutorado PGIE, 2004.

Blanco, A.G. La exposición, un médio de comunicación. Madrid: Alral, 1997.

Cabral, M.(2002) Educação em Museus como produto: Quem está comprando? In: CONFERÊNCIA DE NAIROBI, 2002. Anais... Contribuição para o documento conjunto do CECA-Brasil, n. 1.

Carter, G. The Wider Role of Museum Educators. Committee for Education and Cultural Action (ICOM/CECA) - Study Series, France, p.3-5, oct. 1996.

Cazelli, S. et al. (1998) Aprendizagem compartilhada em museus interativos de ciência. Cadernos de Memória: Museu em transformação, Rio de Janeiro, n.4, ano 6, p. 128-132.

Chadwick, J. A (1999) Survey of Characteristics and Patterns of Behavior in Visitors to a Museum WebSite. In: D. Bearman and J. Trant (Eds.) MUSEUMS AND THE WEB99, Archives and Museum Informatics, 1999, New Orleans. Proceedings... New Orleans: Archives and Museum Informatics. p. 154-162.

Costa, A. C. R. Comunidades Virtuais [mensagem pessoal]. Mensagem recebida por $<$ rocha@atlas.ucpel.tche.br> em 10 ago. 2004.

Fernández, L. A. (1993) Museología - Introducción a La Teoria Y Práctica Del Museo. Ediciones Istmo, España, p. 424

Mansur, O.M.F. C. e Moretto, R. (2000) Aprendendo a ensinar. São Paulo: Elevação. $106 \mathrm{p}$.

Miranda, R. M. (2004) GROA: Um Sistema de Gerência de Repositórios de Objetos de Aprendizagem. Porto Alegre, PPGC/UFRGS. (Dissertação de Mestrado).

Palloff, R.M. e Pratt, K. Construindo Comunidades de Aprendizagem no Ciberespaço: Estratégias eficientes para salas de aula on-line. Trad. Vinícius Figueira. Porto Alegre: Artmed, 2002. 248p.

Payne, M. e Peacock, D. (2004) Collection Object to Learning Object. In: D. Bearman and J. Trant (Eds.) MUSEUMS AND THE WEB 04, 2004, USA. Proceeding... USA: Archives and Museum Informatics.

Schelemmer, E. Projetos de Aprendizagem baseados em problemas: uma metodologia interacionista/construtivista para formação de comunidades em ambientes virtuais de aprendizagem. Colabora - Revista Digital da CVA - RICESU, Curitiba, v.1, n.1, p. 4-11, ago. 2001.

Souza, R.R. Aprendizagem Colaborativa em Comunidades Virtuais, 2000. Dissertação (Mestrado em EP) - PPEP, UFSC, Florianópolis, 2000. 
Trant, J. , Bearman, D.. (2002) Educational Use of Museum Multimedia the AMICO library. Art Libraries Journal. 27 (2). 